\title{
Dynamic response of machine foundation resting on sand- granulated tyre rubber mixtures
}

\author{
Falah Hassan ${ }^{1, *}$ and Salwan Ahmed ${ }^{1}$ \\ ${ }^{1}$ Building and Construction Engineering Department, University of Technology, Baghdad, Iraq
}

\begin{abstract}
This paper examines the dynamic response of machine foundation resting on sand mixed with granular rubber of sizes (0.07-3) $\mathrm{mm}$ to improve the vibration attenuation and to reduce the settlement of the foundation. Granular rubber used is the product of hashing scrap tyres to small pieces in a tyre factory of Babylon in Iraq. Forty Model tests were carried out in a steel box of size (1200x1200x900) mm using a steel square footing of size $(200 \times 200 \times 20) \mathrm{mm}$ and a vertically acting rotating-mass type mechanical oscillator to apply a harmonic vertical mode of vibration. Karbala sand was used in this study with two relative densities (loose and medium) mixed with granular rubber ratios of (4\%,8\% and $12 \%$ ) of dry sand for different depths of mixing of $(0.25 \mathrm{~B}, 0.5 \mathrm{~B}$ and $1 \mathrm{~B})$. The effect of relative density, frequency, depth of mixing and mixing ratio were studied. The results showed that the displacement amplitude and the settlement of machine foundation resting on dry sand granular rubber mixtures decreased with increasing mixing ratio and mixing depth. The optimum mixing ratio and mixing depth for loose state are $(8 \%$ and $0.5 \mathrm{~B})$ at $69 \mathrm{~Hz}$ frequency while they are $(12 \%$ and $1 \mathrm{~B})$ at $80 \mathrm{~Hz}$ frequency. For medium state they are $(8 \%$ and $1 \mathrm{~B}$ ) for both frequencies. The settlement became constant after few seconds of vibration application when the foundation resting on sand mixed with optimum granular rubber content.
\end{abstract}

\section{Introduction}

The volume of used tyre rubbers in the world is significantly increasing every year due to the developing industry and a growing population and their disposals, therefore, they become a major environmental problem worldwide. The large number of scrap tyres is either dumped in landfills or stockpiled across the landscape in huge volume $[1,2,3]$. Tyres in landfills are problematic because they take up large amount of space, which are not compatible, and can 'float' to the top, potentially damaging or breaking the landfill cap $[1,4]$.

The use of tyre shreds or granular rubber recycling from waste tyres in construction projects, such as embankment roads and improvement the soil under foundation may be feasible as an alternate way to consume the huge stockpile of scrap tyre, with a better understanding of the behavior of rubber-soil mixture. Use of these waste materials in different applications can provide an alternative way to consume it from all over the world and consequently can be cost-effective and reduce some environmental problems from waste tyres and give these wastes an ecological value [3].

The rubber-soil mixture was reported by various researchers [4, 5, 6, 7]. They showed that recycled rubber mixed with soil is a potential composite material, which can be advantageously employed in improving the dynamic behavior of new composite materials, particularly in the case of roads highway and embankments.

A laboratory model test of tyre chips embankment was conducted to generate deformation response data of various constructions under repetitive loads. A better performance in decreasing the surface plastic displacement was noticed, when the tyre-chips covered by a relatively thick soil cap compared to using the tyrechips in the whole of the fill [5].

The dynamic properties (shear modulus and damping ratio) of specimens constructed by different percentages of granulated tyre rubber and Ottawa sand using torsional resonant column test, were strongly influenced by the percentages of rubber. The shear modulus decreases and the damping ratio increase with increasing the rubber content [7].

The behavior of recycled materials (tyre buffing's) for highways under static and dynamic loads was investigated. The tire buffing may be used to modify the strength, deformation and dynamic behavior of sand and their use is economically feasible [6].

The maximum load carrying capacity associated with less value of rebound deflection of flexible pavement on expansive soil subgrade was obtained for rubber mixed with gravel subbase compared to rubber mixed with fly ash subbase [8].

This paper aims to study the dynamic response of machine foundation resting on sand-granulated tire

\footnotetext{
* Corresponding author: ffhhhzmr@yahoo.com
} 
rubber mixtures through study the vibration amplitude and settlement of the foundation and compare the results with that of the soil alone. Also the use of the granular tyre rubber in this field has another benefit contribution solving the problem of the pollution.

\section{Materials used}

\subsection{Sand}

Karbala sand is used in the present study. Standard tests are performed to determine the physical properties. The details of these properties are listed in Table (1). The grain size distribution is shown in Figure (1). The soil is classified as poorly graded sand (SP) according to the unified soil classification system.

Table 1. Physical properties of sand used

\begin{tabular}{|c|c|c|}
\hline Property & Value & Specification \\
\hline $\begin{array}{c}\text { Specific gravity, } \\
\text { Gs }\end{array}$ & 2.64 & ASTM D854 [9] \\
\hline $\begin{array}{l}\text { Coefficient of } \\
\text { curvature, } \mathrm{Cc}\end{array}$ & 0.87 & \multirow{4}{*}{$\begin{array}{l}\text { ASTM D422-63 } \\
\text { and } \\
\text { ASTM D2487-06 } \\
{[10,11]}\end{array}$} \\
\hline $\begin{array}{l}\text { Coefficient of } \\
\text { uniformity, } \mathrm{Cu}\end{array}$ & 2.37 & \\
\hline $\begin{array}{c}\text { D10, D30, D60 } \\
(\mathrm{mm})\end{array}$ & $0.16,0.23,0.38$ & \\
\hline USCS-soil type & SP & \\
\hline $\begin{array}{l}\text { Maximum dry } \\
\text { unit weight, } \\
\gamma d \max , \mathrm{kN} / \mathrm{m} 3\end{array}$ & 18.82 & $\begin{array}{c}\text { ASTM D4253-0 } \\
{[12]}\end{array}$ \\
\hline $\begin{array}{l}\text { Minimum dry } \\
\text { unit weight, } \\
\gamma \mathrm{dmin}, \mathrm{kN} / \mathrm{m} 3\end{array}$ & 15.32 & $\begin{array}{c}\text { ASTM D4254-00 } \\
{[13]}\end{array}$ \\
\hline
\end{tabular}

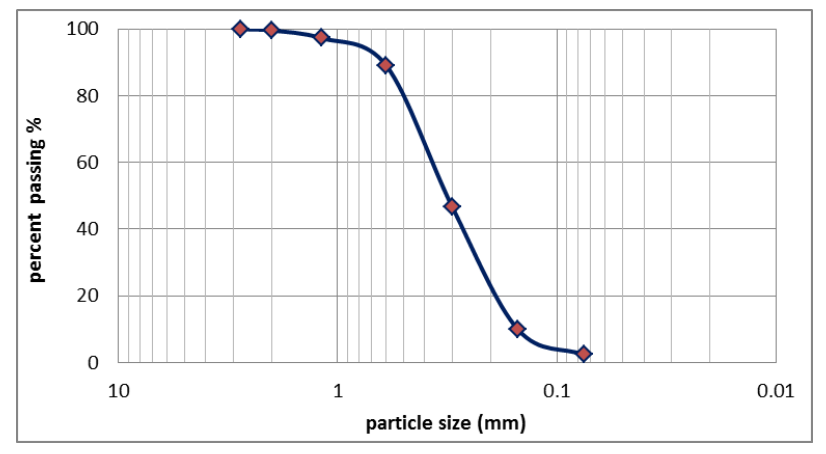

Fig.1. Grain size distribution of Karbala Sand

\subsection{Granular rubber}

Granular rubber used is the product of hashing scrap tyres to small pieces in a tyre factory of Babylon in Iraq. The engineering properties of tire rubber are listed in Table (2), these properties are taken from the tyre factory. The grain size distribution of granulated rubber is shown in Figure (2). The specific gravity of this material is determined according by ASTM D 854, and its value 0.88. According to ASTM D6270-98 and since the grain size of the waste tyre rubber is $(0.07-3) \mathrm{mm}$, the rubber is specified as the granular rubber.

Table 2. Engineering properties of tire rubber

\begin{tabular}{|c|c|c|}
\hline Property & Value & Specification \\
\hline \multirow{2}{*}{ Void ratio, e } & $\begin{array}{c}1.5-2.5 \\
\text { Uncompacted }\end{array}$ & \\
\cline { 2 - 2 } & $\begin{array}{c}0.9-1.2 \\
\text { Compacted }\end{array}$ & \multirow{2}{*}{ ASTM } \\
& $1241-5171 \mathrm{kPa}$ & D6270-98 \\
Elastic Modulus, E & 0.5 & {$[14]$} \\
\hline Poisson's Ratio, $\mu$ & $2 \%-4 \%$ & \\
\hline $\begin{array}{c}\text { Water Absorption } \\
\text { Capacity }\end{array}$ & & \\
\hline
\end{tabular}

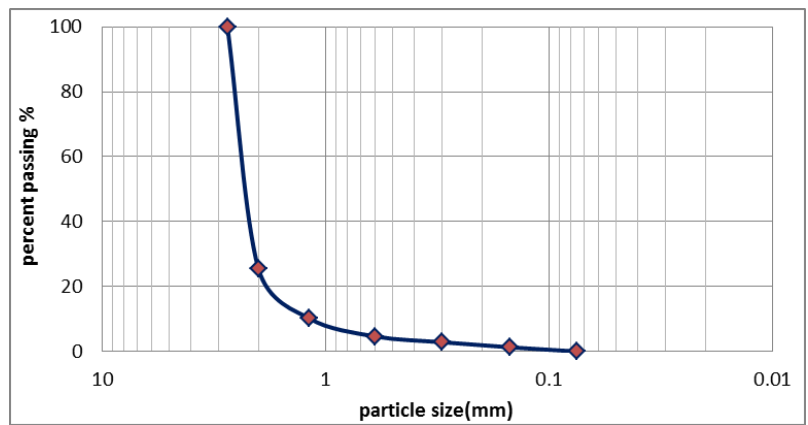

Fig.2. Grain size distribution of granular rubber

\section{Model preparation and setup used}

A steel container of size $(1200 \times 1200 \times 900) \mathrm{mm}$ is used for preparation of sand. A predetermined weight of sand is poured in a testing tank in layers of $100 \mathrm{~mm}$ thick. Each layer is compacted to maintain a uniform density throughout the model and to achieve the desired relative densities (35\% and 60\%). To study the effect of granular rubber in depth, three depths of sand-granulated rubber of $(0.25 \mathrm{~B}, 0.5 \mathrm{~B}$ and $1 \mathrm{~B})$ are used, where $\mathrm{B}$ is the width of the footing. The percentages of granulated rubber by weight of sand are $(4 \%, 8 \%$ and $12 \%)$. The final layer is leveled and then steel square footing of size $(200 \times 200 \times 20) \mathrm{mm}$ fixed with the mechanical oscillator on the top of the footing is placed carefully in the center of the tank as shown in Plate (1). A piezoelectric type vibration pickup is placed on the top of the footing to measure the displacement amplitude and connected to a data acquisition system. LVDT is placed on the edge of the footing to measure the settlement of the foundation. A speed control unit is connected to the oscillator to achieve the desired frequencies $69 \mathrm{~Hz}$ and $80 \mathrm{~Hz}$.

\section{Testing program and testing procedure}

Forty model tests are performed. They are divided into 20 tests for each relative density (35\% and 60\%). Two of which are carried out on the sand without granular 
rubber for comparison. Three percentages of granular rubber by weight of sand are used $(4 \%, 8 \%$, and $12 \%)$ for the soil under the footing at a depth of $(0.25 \mathrm{~B}, 0.5 \mathrm{~B}$ and $1 \mathrm{~B}$ ) from the footing. For each relative density two frequencies are used $69 \mathrm{~Hz}$ and $80 \mathrm{~Hz}$. After the model preparation a harmonic load was applied at the foundation using a mechanical oscillator having a maximum speed of $6500 \mathrm{rpm}$. The operation is continued to achieve a stable reading. The results of displacement amplitude and settlement of the foundation are recorded with time on a PC connected to a data acquisition system.
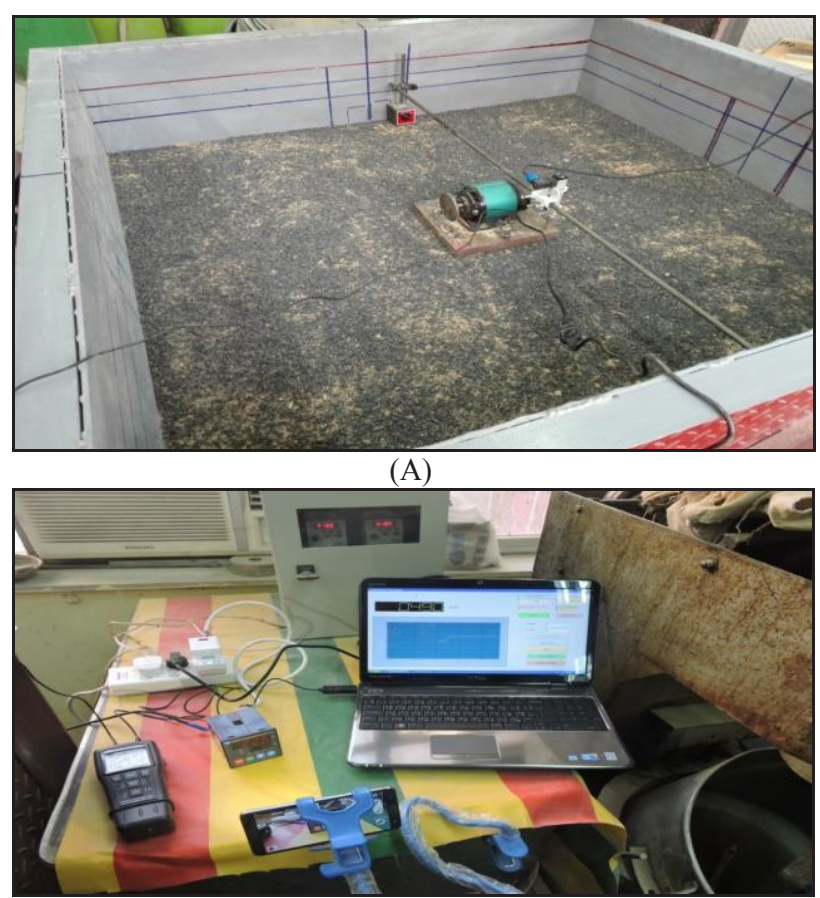

(B)

Plate.1. (A) Model setup and test apparatus (B) Data acquisition system

\section{Results and discussion}

The results of experimental study are presented in this section. The presentation focusses on the effect of two main parameters, mixing ratio of granular rubber and the depth of an improved layer on displacement amplitude and settlement of machine foundation for the two relative densities under the effect of the two frequencies $69 \mathrm{~Hz}$ and $80 \mathrm{~Hz}$.

\subsection{Effect of mixing ratio (R.D=35\%)}

\subsubsection{Displacement amplitude}

The variation of displacement with time was measured for each test by using a vibration meter; where-as the displacement amplitude (Az) was recorded at the steady state, which occurred after (500) sec from the operation of the oscillator.
The variation of displacement with time for $69 \mathrm{~Hz}$ frequency and $(0.25 \mathrm{~B})$ depth of mixing is shown in Figure (3). It can be noticed from this figure that the displacement of sand is more than the displacement of sand granular rubber mixture for different ratios of mixing. The optimum mixing ratio at this depth of mixing that give the minimum value of $(\mathrm{Az})$ is found at the mixing ratio $(8 \%)$, beyond which the value of $(\mathrm{Az})$ decreases.

Figure (4) demonstrates the value of ( $\mathrm{Az}$ ) with mixing ratio and depth of mixing for $69 \mathrm{~Hz}$ frequency. It is clear from the figure that the best reduction occurred at $(0.5 \mathrm{~B})$ mixing depth for $(8 \%)$ mixing ratio.

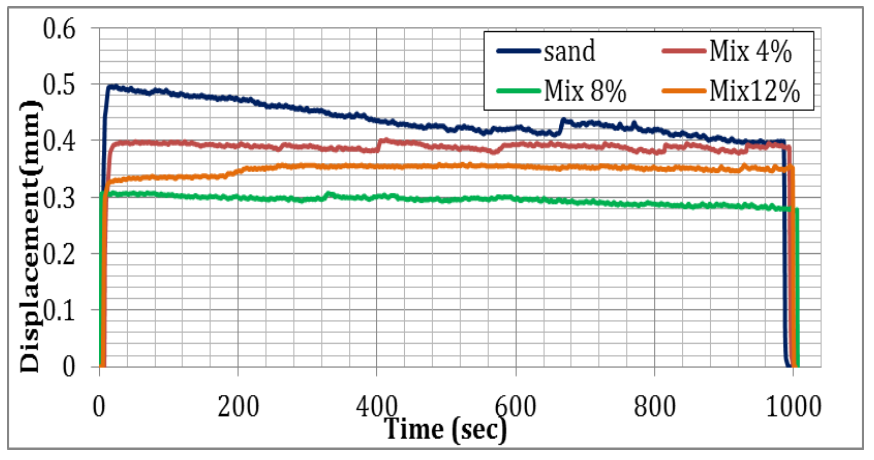

Fig.3. The relationship between displacement and time for $\mathrm{fr}$ $69 \mathrm{~Hz}$ and depth of mixing $0.25 \mathrm{~B}$

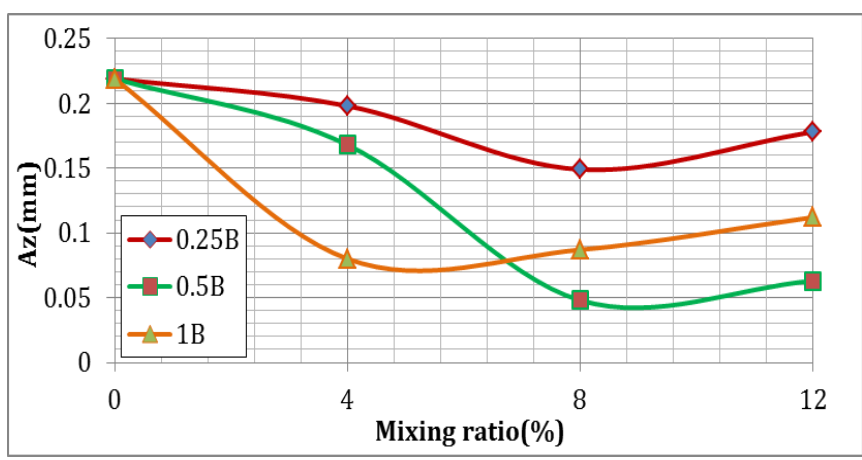

Fig.4. Variation of displacement amplitude (Az) with mixing ratios for different depths of mixing and fr $69 \mathrm{~Hz}$

The results of displacement for $(0.25 \mathrm{~B})$ depth of mixing and $80 \mathrm{~Hz}$ frequency are shown in Figure (5). The addition of granular rubber decreases the displacement and the best ratio is (12\%). Figure (6) shows the variation of $(\mathrm{Az})$ with a mixing ratio for different depth of mixing. It is clear from the figure that the best mixing ratio is $(12 \%)$ at a depth of mixing (1B).

The above results showed that the optimum mixing ratio increases with an increase in frequency. 


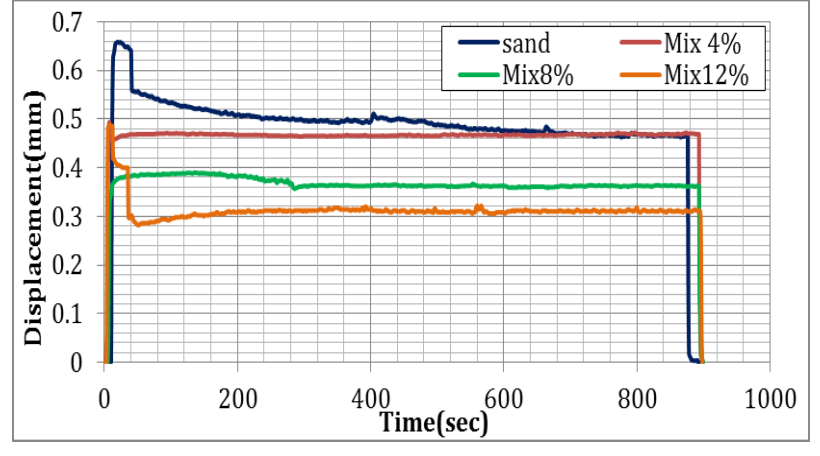

Fig.5. The relationship between displacement and time for fr $80 \mathrm{~Hz}$ and depth of mixing $0.25 \mathrm{~B}$.

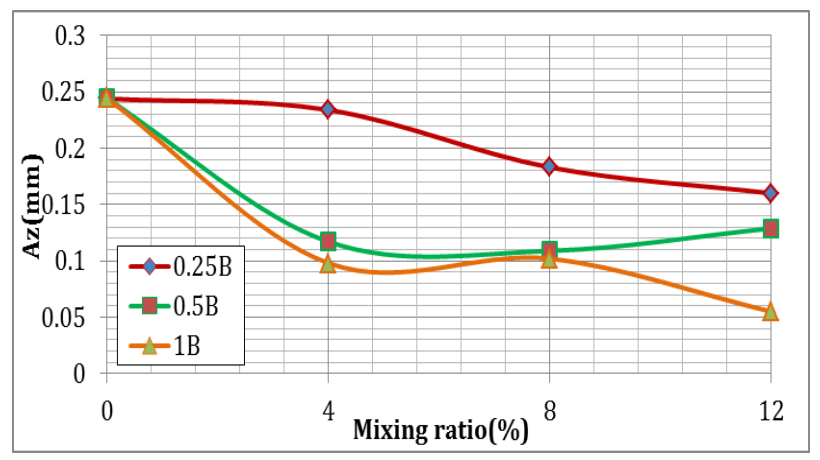

Fig.6. Variation of displacement amplitude (Az) with mixing ratios for different depths of mixing and fr $80 \mathrm{~Hz}$.

\subsubsection{Settlement of foundation}

The variation of settlement with time for the different mixing ratios and $69 \mathrm{~Hz}$ frequency is shown in Figure (7). It is clear from the figure that the settlement decreases with increase of mixing ratio and after a short time of vibration application the value of settlement becomes constant, when the mixing ratio is equal to or greater than $(8 \%)$. This means that the use of optimum percent of granular rubber stopped the plastic deformation of the sand under the foundation.

Figure (8) illustrates the variation of a final settlement ( $\mathrm{Stf}$ ) with a mixing ratio for $69 \mathrm{~Hz}$ frequency and different depths of mixing. It can be observed that the settlement decreases with increasing mixing ratio and the best values of mixing ratio is $(12 \%)$.

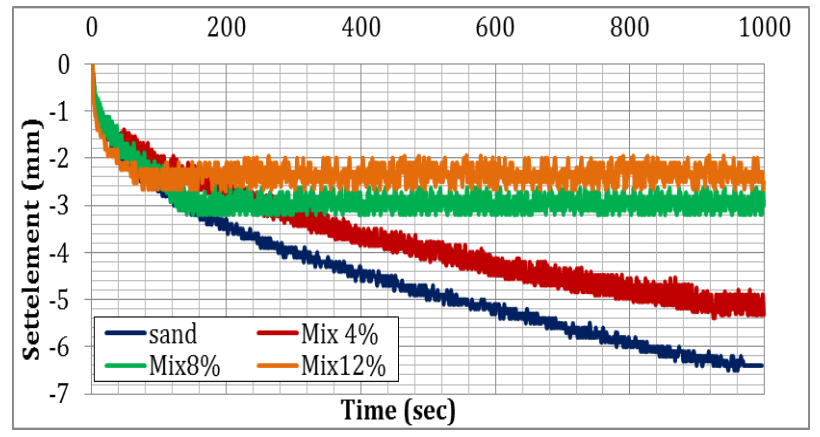

Fig.7. The relationship between settlement and time for fr 69 $\mathrm{Hz}$ and depth of mixing $0.25 \mathrm{~B}$.

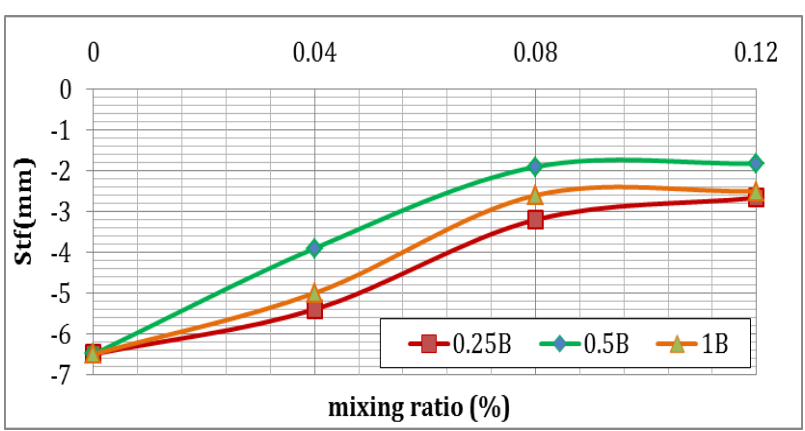

Fig.8. Variation of settlement with time for fr $69 \mathrm{~Hz}$ and depth of mixing $0.25 \mathrm{~B}$.

The results of settlement for the $80 \mathrm{~Hz}$ frequency, (0.25B) mixing depth and different mixing ratios are shown in Figure (9). It is clear that the plastic deformation decreases remarkably when using the rubber and the settlement stops after a very short time from the vibration application.

Figure (10) demonstrates the variation of settlement with a mixing ratio for different depths of mixing. The settlement decrease with an increase in mixing ratio.

From the above results it can be concluded that the settlement increases with increase in frequency for the same mixing ratio.

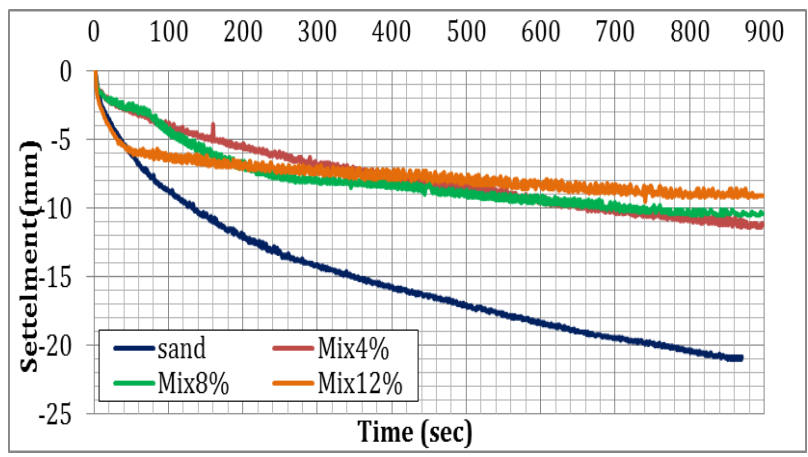

Fig.9. The relationship between settlement and time for fr 80 $\mathrm{Hz}$ and depth of mixing $0.25 \mathrm{~B}$.

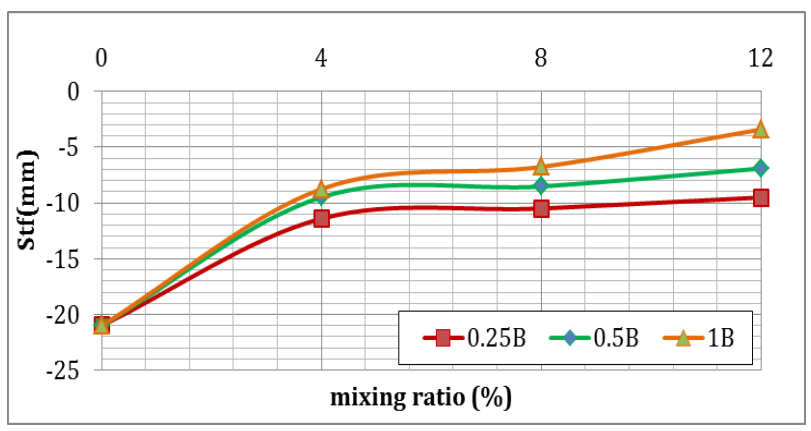

Fig.10. Variation of settlement with time for fr $80 \mathrm{~Hz}$ and depth of mixing $0.25 \mathrm{~B}$. 


\subsection{Effect of mixing depth (R.D=35\%)}

\subsubsection{Displacement amplitude}

The relationship between displacement amplitude (Az) and the depth of mixing for different ratios are shown in Figures (11) and (12) for frequencies $69 \mathrm{~Hz}$ and $80 \mathrm{~Hz}$, respectively. It is clear that the best depth is $(0.5 \mathrm{~B})$ for both frequencies.

\subsubsection{Settlement of foundation}

The variation of settlement with depth of mixing for 69 $\mathrm{Hz}$ and $80 \mathrm{~Hz}$ frequencies are shown in Figure (13) and Figure (14), respectively. The best depth for controlling the settlement is $(0.5 \mathrm{~B})$ for the $69 \mathrm{~Hz}$ frequency, while the settlement decreases with increasing depth of mixing for $80 \mathrm{~Hz}$ frequency.

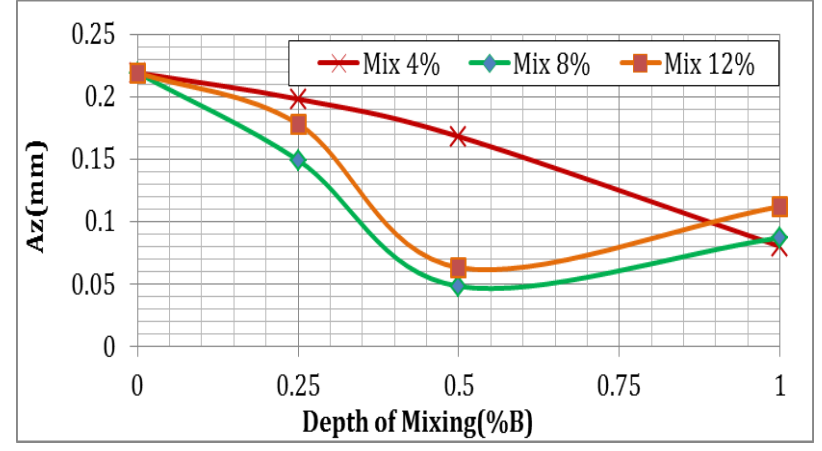

Fig.11. Variation of Az with depths of mixing for different mixing ratios and fr $69 \mathrm{~Hz}$.

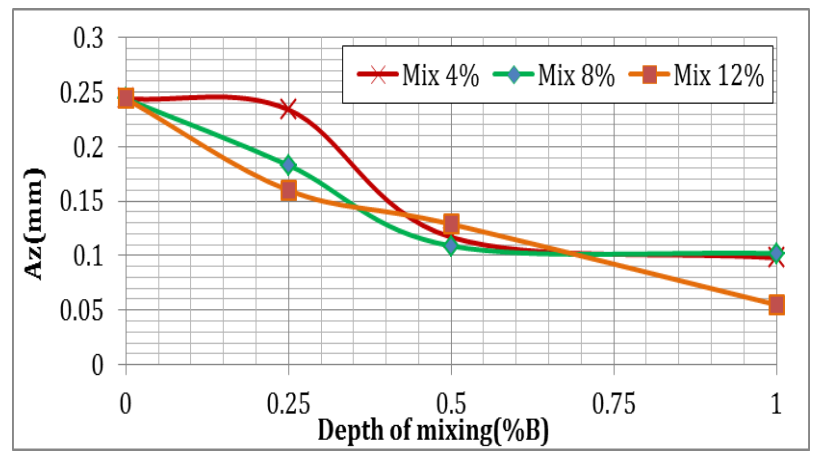

Fig.12. Variation of Az with depths of mixing for different mixing ratios and fr $80 \mathrm{~Hz}$.

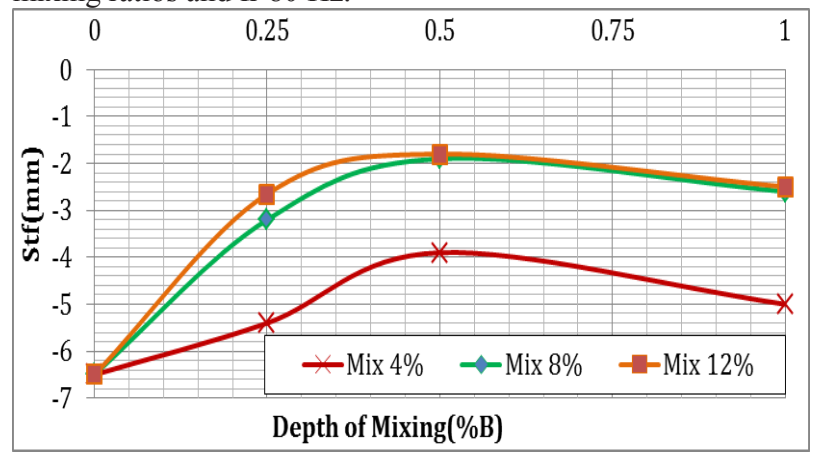

Fig.13. Variation of Stf with depths of mixing for different mixing ratios and fr $69 \mathrm{~Hz}$.

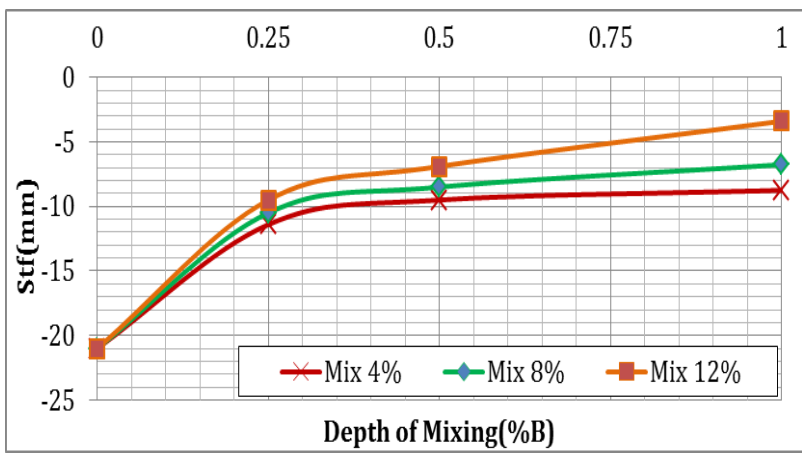

Fig.14. Variation of Stf with depths of mixing for different mixing ratios and fr $80 \mathrm{~Hz}$.

\subsection{Effect of mixing ratio (R.D $=60 \%)$}

\subsubsection{Displacement amplitude}

Figure (15) represents the relationship between displacement and time for $(0.25 \mathrm{~B})$ depth of mixing and for different mixing ratios. It is clear from the figure that the best mixing ratio is (4\%) and the displacement amplitude stays nearly constant for the ratios above $(4 \%)$, while the best value of the ratio is $(12 \%)$ when the frequency increases to $80 \mathrm{~Hz}$ for the same depth as shown in Figures (17) and (18).

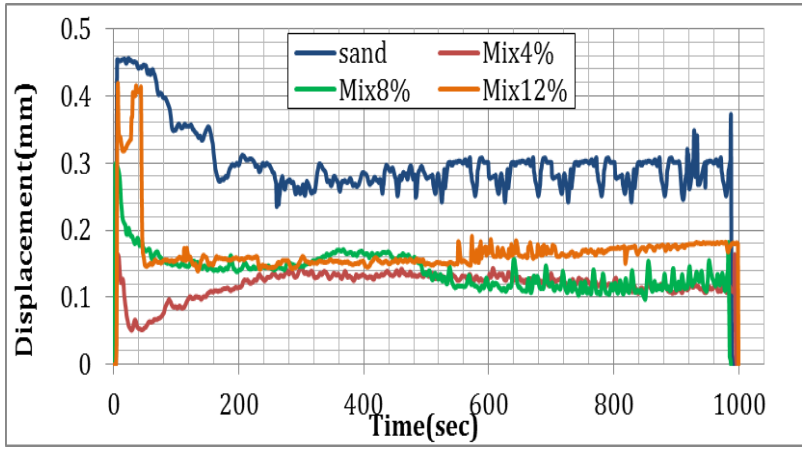

Fig.15. The relationship between displacement and time for fr $69 \mathrm{~Hz}$ and depth of mixing $0.25 \mathrm{~B}$.

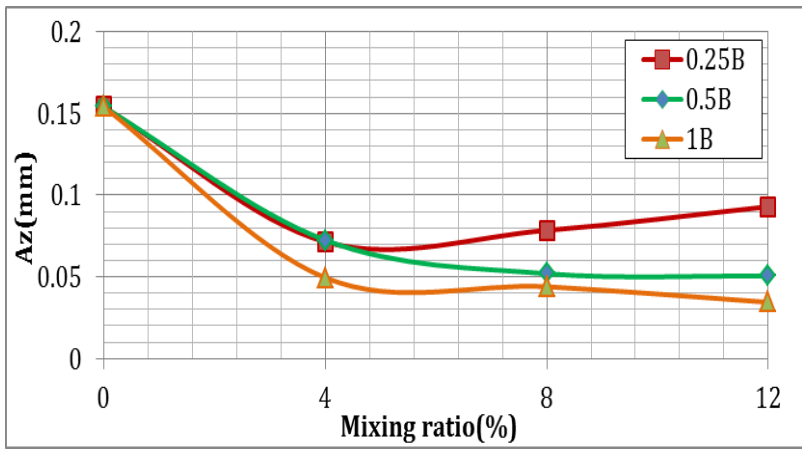

Fig.16. Variation of displacement amplitude with mixing ratios for each depth of mixing and fr $69 \mathrm{~Hz}$ 


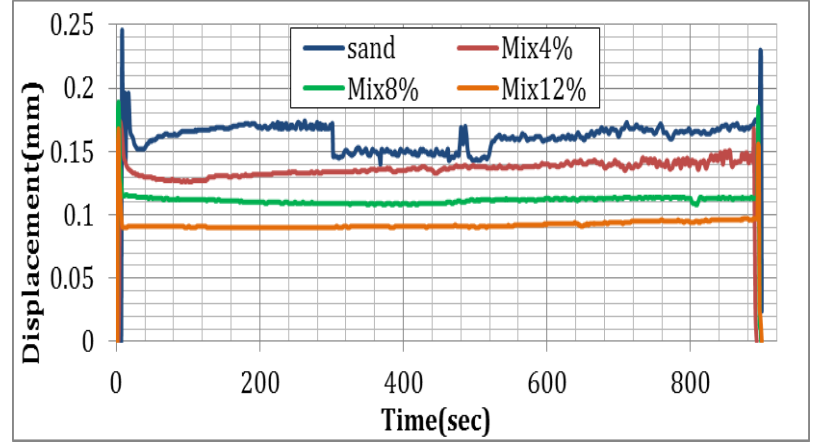

Fig.17. The relationship between displacement and time for fr $80 \mathrm{~Hz}$ and depth of mixing $0.25 \mathrm{~B}$

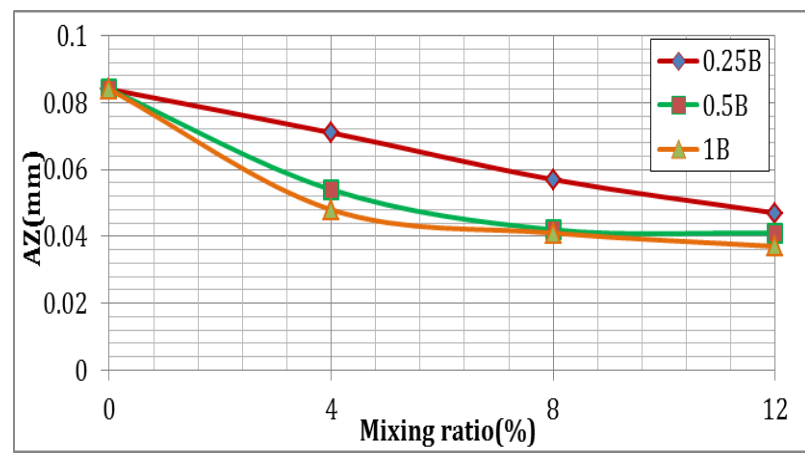

Fig.18. Variation of displacement amplitude with mixing ratios for each depth of mixing and fr $69 \mathrm{~Hz}$.

The above results showed that the displacement amplitude for medium sand is less than that of loose state under the same condition, due to increase in the stiffness of sand.

\subsubsection{Settlement of foundation}

The relationship of settlement with time for $(0.25 \mathrm{~B})$ depth of mixing and $69 \mathrm{~Hz}$ frequency and different mixing ratios is shown in Figure (19). The settlement is more uniform compared to the same models of relative density (35\%). The results of settlement increase with an increasing mixing ratio until (8\%) then become constant as shown in Figure (20).

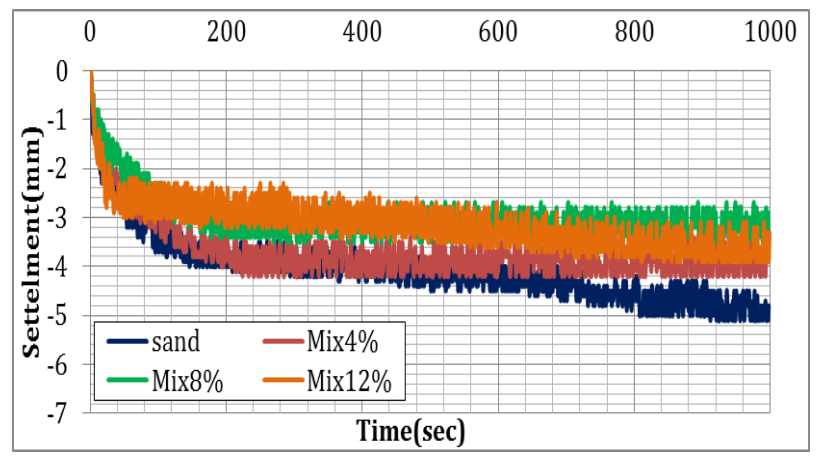

Fig.19. The relationship between settlement and time for fr 69 $\mathrm{Hz}$ and mixing depths $0.25 \mathrm{~B}$

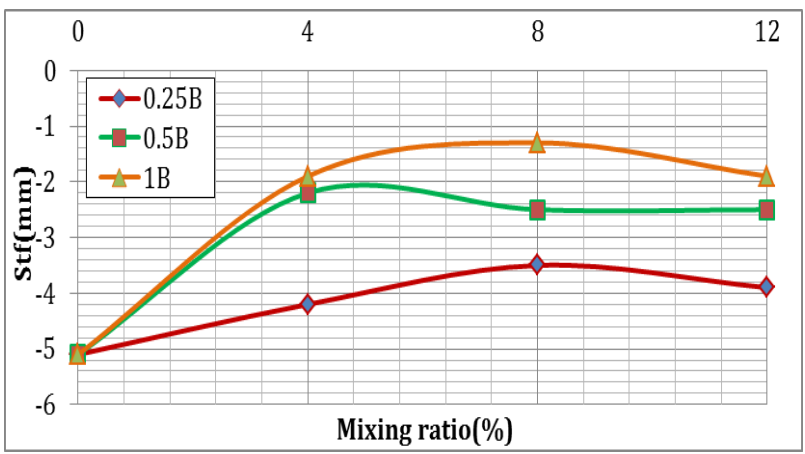

Fig.20. Variation of final settlement with mixing ratios for each depth of mixing and fr $69 \mathrm{~Hz}$

In the same way, the results of the settlement with time for frequency of $(80 \mathrm{~Hz})$ is presented in Figure (21) for depth of mixing $(0.25 \mathrm{~B})$. It is clear that the values of settlement stays constant after a very short time from the operation of the machine. Also the settlement decreases with increase in mixing ratio until $(8 \%)$, then becomes constant as shown in Figure (22). This means that the effect of frequency decreases with increase in the relative density.

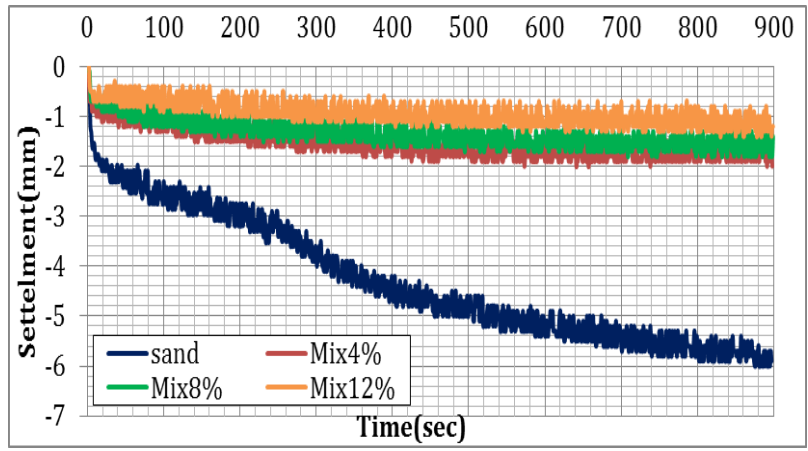

Fig.21. The relationship between settlement and time for fr 80 $\mathrm{Hz}$ and mixing depths $0.25 \mathrm{~B}$

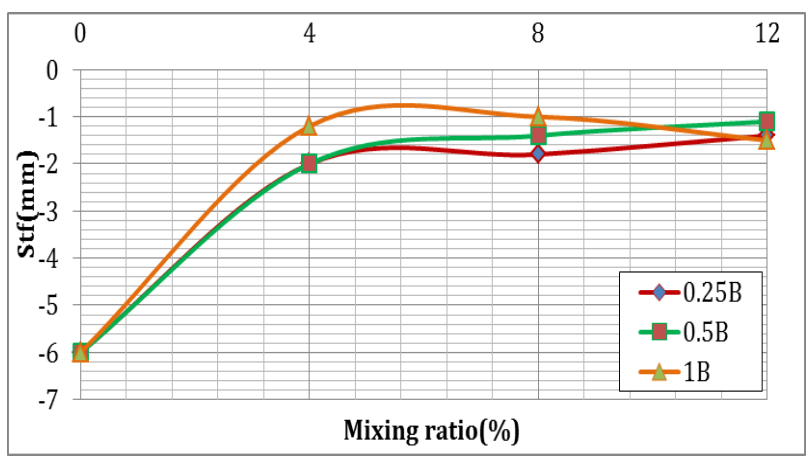

Fig.22. Variation of final settlement with mixing ratios for each depth of mixing and fr $80 \mathrm{~Hz}$

\subsection{Effect of mixing depth (R.D $=60 \%)$ :}

\subsubsection{Displacement Amplitude}


The variation of displacement amplitude $(\mathrm{Az})$ with a depth of mixing under the effect of $69 \mathrm{~Hz}$ and $80 \mathrm{~Hz}$ frequencies for different mixing ratios are shown in Figure (23) and (24). It is clear from the figures that the best depth of improvement is $(0.5 \mathrm{~B})$ for the both frequencies.

\subsubsection{Settlement of foundation}

the results of the settlement with mixing depth for the two frequencies are shown in Figures (25) and (26). In general the settlement decrease with increase in the depth of mixing and frequency, but for sand without rubber the settlement increases with increase in the frequency. The preferred depth of mixing layer is (1B) for both frequencies, since at this depth the final settlement value is the minimum.

When mixing the granular rubber with sand, the settlement becomes approximately constant with time and the mixture behaves as an elastic material.

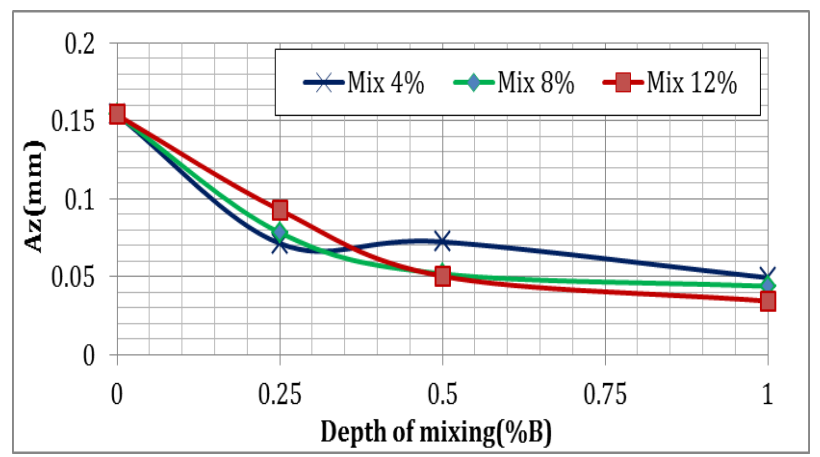

Fig.23. Variation of Az with depths of mixing for different mixing ratios and fr $69 \mathrm{~Hz}$

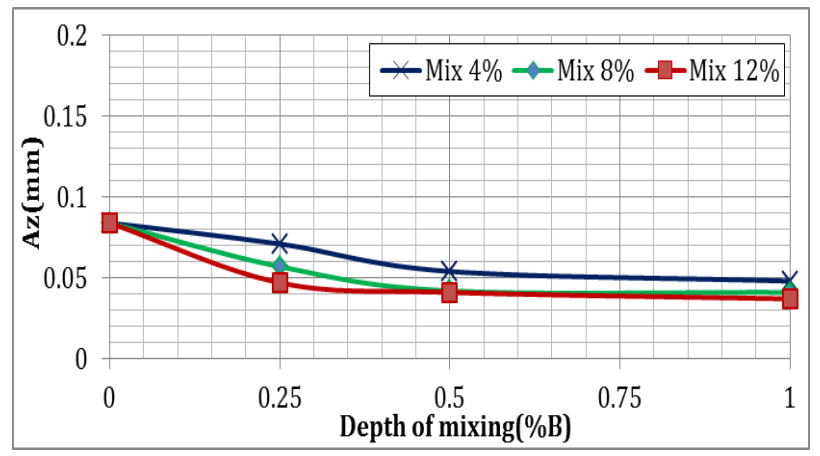

Fig.24. Variation of Az with depths of mixing for different mixing ratios and $\mathrm{fr} 80 \mathrm{~Hz}$

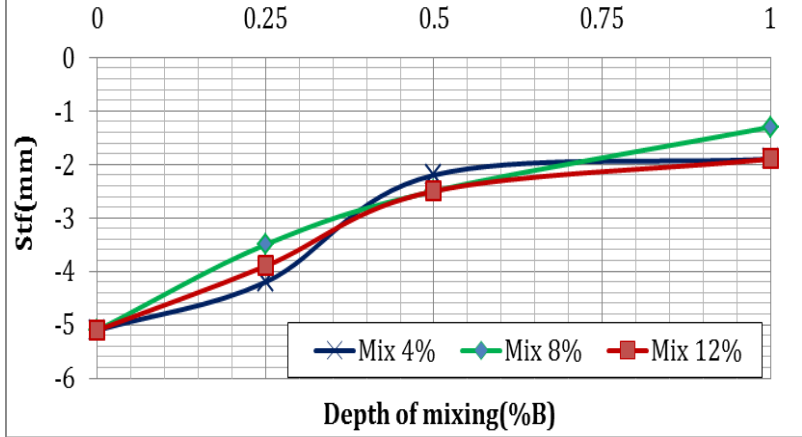

Fig.25.Variation of (Stf) with depths of mixing for different mixing ratios and fr $69 \mathrm{~Hz}$.

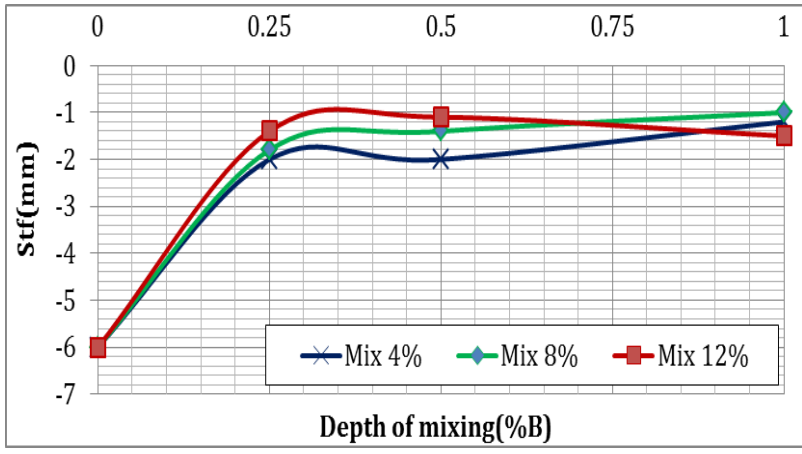

Fig.26.Variation of (Stf) with depths of mixing for different mixing ratios and fr $80 \mathrm{~Hz}$.

\section{Conclusions}

- For dry loose sand-granular rubber mixtures, the displacement amplitude increases with increase in the frequency of the machine, and decreases with increase in the mixing ratio and depth of mixing. The optimum mixing ratio is $(8 \%)$ and the best depth of mixing is $(0.5 \mathrm{~B})$ for the $69 \mathrm{~Hz}$ frequency, while the optimum mixing ratio is $(12 \%)$ and the best depth of mixing is $(1 \mathrm{~B})$ for the $80 \mathrm{~Hz}$ frequency.

- For dry medium sand-granulated rubber mixtures, the displacement amplitude decrease with increasing frequency, increasing mixing ratio and depth of mixing. The optimum mixing ratio is $(8 \%)$ and the preferred mixing depth is $(1 \mathrm{~B})$.

- For dry loose sand-granular rubber mixtures, the final settlement increases with increase in frequency and decrease with increase in mixing ratio and depth of mixing. The optimum mixing ratio and mixing depth are $(8 \%$ and $0.5 \mathrm{~B})$ for frequency $69 \mathrm{~Hz}$, while they are $(12 \%$ and $1 \mathrm{~B})$ for the $80 \mathrm{~Hz}$ frequency.

- For dry medium sand-granulated rubber mixtures, the settlement decreases with increase in mixing ratio and mixing depth. The optimum mixing ratio and depth of mixing are $(8 \%$ and $1 \mathrm{~B})$ for the two frequencies. 
- For both relative densities used, the most effective depth of mixing is (1B) and the preferred mixing ratio is $(12 \%)$.

- The settlement of the foundation resting on sand mixed with optimum granular tyre rubber ratio becomes approximately constant after a short time of machine vibration and the mixture behaves as an elastic material .

\section{References}

1. Cetin, H., Fener, M. And Gunaydin, O. Eng. Geol. 88(1-2), 110-120, (2006).

2. Chiu, C.T. J.Res. Conserv. Recy. 52(3), 522-532, (2008).

3. Wu, J.Y. And Tsai, M.,Waste. Manage. 29(2) ,636642, (2009).

4. Lee, J., Dodds, J. And Santamarina, J.C., J. Mater. Civil.Eng. - ASCE, 19(2), 179-184, (2007).

5. Bosscher, P.J., Edil, T.B. and Kuraoka, S., J.Geotech. Geoenviron. Eng. - ASCE, 123(4), 295-304, (1997).

6. Edincliler, A., Baykal, G. and Dengili, K., Resour. Constr. Recy., 42(3), 223-237, (2004).

7. Feng, Z.Y. and Sutter, K.G., Geotech. Test.J., 23(3), 338-344, (2000).

8. Prasad, D.S.V. and Prasada Raju, G.V.R., ARPN. J. Eng. Appl. Sci., 4(6), 89-92, (2009).

9. American Society of Testing and Materials (ASTM).ASTMD854,WestConshohocken,

Pennsylvania, USA, (2006).

10. American Society of Testing and Materials (ASTM). ASTM D422-63 (2002), West Conshohocken, Pennsylvania, USA, (2006).

11. American Society of Testing and Materials (ASTM).ASTM D2487-06, West Conshohocken, Pennsylvania, USA, (2006).

12. American Society of Testing and Materials (ASTM). ASTM D4253-00 (2006), West Conshohocken, Pennsylvania, USA, (2006).

13. American Society of Testing and Materials (ASTM). ASTM D4254-00 (2006), West Conshohocken, Pennsylvania, USA, (2006).

14. American Society of Testing and Materials (ASTM).ASTM D6270-98, West Conshohocken, Pennsylvania, USA ,P.40-63, (2004). 